\title{
Insulin Influences Autophagy Response Distinctively in Macrophages of Different Compartments
}

\author{
Karen K. S. Sunahara ${ }^{a, b}$ Fernanda P. B. Nunes ${ }^{a}$ Marisa A. P. Baptista ${ }^{c}$ Carina Strell $^{d}$ \\ Paulina Sannomiya ${ }^{\mathrm{e}}$ Lisa S. Westerberg ${ }^{c}$ Joilson O. Martins ${ }^{\mathrm{a}}$ \\ aLaboratory of Immunoendocrinology, Department of Clinical and Toxicological Analyses, Faculty of \\ Pharmaceutical Sciences of University Sao Paulo (FCF/USP), São Paulo, 'Experimental Physiopathology, \\ Department of Sciences/Experimental Physiopatholgy, Medical School, University of São Paulo, Brazil; \\ 'Department of Microbiology Tumor and Cell biology, Karolinska Institutet, Stockholm, 'Department \\ of Oncology Pathology, Karolinska Institutet, Stockholm Sweden; eInstitute of Heart (InCor), LIM-11, \\ University of São Paulo Medical School, São Paulo, Brazil
}

\section{Key Words}

Insulin - Diabetes Mellitus - Alveolar macrophages - Innate immunity • Autophagy • Bone Marrow Macrophages $•$ Macrophages polarization

\begin{abstract}
Background/Aims: Diabetes mellitus (DM) is characterized by hyperglycemia, associated to a lack or inefficiency of the insulin to regulate glucose metabolism. DM is also marked by alterations in a diversity of cellular processes that need to be further unraveled. In this study, we examined the autophagy pathway in diabetic rat macrophages before and after treatment with insulin. Methods: Bone marrow-derived macrophages (BMM), bronchoalveolar lavage (BAL) and splenic tissue of diabetic male Wistar rats (alloxan, $42 \mathrm{mg} / \mathrm{kg}$, i.v., 10 days) and control rats (physiological saline, i.v.). Some diabetic rats were given neutral protamine Hagedorn insulin (4 IU, s.c.) $8 \mathrm{~h}$ before experiments. For characterization of the model and evaluation of the effect of insulin on the autophagic process, the following analyzes were performed: (a) concentrations of cytokines: interleukin (IL)- $1 \beta$, tumor necrosis factor (TNF)- $\alpha$, IL-6, IL-4, IL-10, cytokine-induced neutrophil chemoattractant (CINC)-1 and CINC-2 in the BAL supernatant was measured by ELISA; (b) characterization of alveolar macrophage (AM) of the BAL as surface antigens (MHCII, pan-macrophage KiM2R, CD11b) and autophagic markers (protein microtubule-associated light chain (LC)3, autophagy protein (Atg) 12 by flow cytometry and confocal microscopy (c) study of macrophages differentiated from the bone marrow by flow cytometry and confocal microscopy (d) histology of the spleen by immunohistochemistry associated with confocal microscopy. Results: Interestingly, insulin exerted antagonistic effects on macrophages from different tissues. Macrophages from bronchoalveolar lavage (BAL) enhanced their LC3 autophagosome bound content after treatment with insulin whereas splenic macrophages from red pulp in diabetic rats failed to enhance their Atg 12 levels compared to control animals. Insulin treatment in diabetic rats did not change LC3 content in bone marrow derived macrophages (BMM). M1 and M2 macrophages behaved accordingly to the host they were derived from. Diabetic M1 BMM had their LC3 vesicle-bound
\end{abstract}


content diminished and M2 BMM enhanced their LC3 levels and insulin treatment failed to rescue autophagy to control levels. Insulin normalizes CINC-2 level but does not modulate autophagy markers. Conclusion: Taking these results together, diabetic macrophages derived from different compartments show different levels of autophagy markers compared to healthy animals, therefore, they suffer distinctively in the absence of insulin.

Copyright (C) 2014 S. Karger AG, Basel

\section{Introduction}

During treatment and follow up of patients with diabetes mellitus (DM), hyperglycemia has for long been an undesirable element. Polyuria, polyphagia and polydipsia, three cardinal symptoms induced by hyperglycemia, have always blurred signs of insulin absence, such as enhanced muscle destruction and lower utilization of glucose by myocytes. Muscle destruction might induce loss of weight, which can normally be observed in type I diabetic patients, since insulin is a key factor for muscle eutrophy maintenance [1]. Moreover, the absence of insulin itself may change other physiologic cell pathways. The internalization of glucose transporters [2], abrogating the availability of glucose intracellularly, is just one of the alterations in cell metabolism induced by lack of insulin. Recently, type I diabetes mellitus (DMI) has been associated to autophagy-lysosome pathway (ALP) dysfunction, which among other defects, contributes for pancreatic $\beta$ cells destruction [3]. Important for cell and tissue homeostasis maintenance, ALP dysfunction has also been associated to other disorders, such as Alzheimer [4], cancer and autoimmunity [5]. Moreover, autophagy has also been associated to microbial resistance. It has been suggested that some pathogens escape host defense, highjack the autophagy pathway and may establish a persistent infection [6]. Infection is of particular concern for DM patients and according to the American Diabetes Association (ADA) website, individuals with diabetes have three times higher risk of dying from flu or pneumonia since they are more susceptible to secondary infections [7]. Styes, folliculitis and other skin complications are also more common in people with diabetes. Experiments using Komeda diabetes-prone (KDP) rats infected with Mycobacterium tuberculosis (Mt), have shown that the burden of the bacilli observed in lungs and spleens of rats was more common when compared to healthy controls. In infected KDP rats, bacteremia impaired the production of nitric oxide (NO) by alveolar macrophages (AM) [8] that constitute an important entry barrier. Studies in alloxan-induced diabetic rats have also shown impairment in peripheral blood leukocyte phagocytic activities $[9,10]$. Using the same model, it was published that diabetic AM have impaired phagocytosis [11]. In patients, diabetic neutrophils have shown dysfunction on their chemotactic and phagocytic abilities [12] and some of these alterations are reversible after insulin treatment [13]. How autophagy and phagocytosis are associated to each other is uncertain. Upon infection, AM from mice lacking autophagy gene 7 (Atg $7^{-/}$) sheltered higher amount of Mycobacterium bovis bacillus Calmette-Guerin (BCG) and Mt compared to normal mice. [14]. After autophagy induction, macrophages phagocyted more BCG, although showing lower survival [15].

In this work, using a well established rat model for type I diabetes, we would like to investigate if there is any difference on autophagy phenotype in macrophages derived from bone marrow, BAL fluid and spleens in diabetic rats. Furthermore, verify if there is any influence of insulin treatment upon autophagy pathway.

\section{Material and Methods}

\section{Animal diabetes model}

Wistar male rats 8 weeks of age, weighing approximately $200 \mathrm{~g}$ at the beginning of the experiments were housed in a $12 \mathrm{~h} / 12 \mathrm{~h}$ light/dark cycle and allowed standard pellet diet and tap water ad libitum throughout the observation period. All experiments were in accordance with the Brazilian National Council 
for the Control of Animal Experimentation (CONCEA) and approved by the Ethical Committee on Animal Use (CEUA) of the Faculty of Pharmaceutical Sciences (FCF) and Medical School (FM), University of São Paulo, before initiating the experiments.

Animals were divided in three groups: control (C), diabetic (D) and diabetic rats treated with insulin (DI) and followed protocol published previously [16]. Briefly, diabetes mellitus was induced by the intravenous injection of $42 \mathrm{mg} / \mathrm{kg}$ of alloxan (ALX) (Sigma Chemical Co, St Louis, Mo) dissolved in physiological saline $(0,9 \% \mathrm{NaCl})$. Control rats were injected with physiological saline alone. Ten days after diabetes induction, peripheral blood glucose (from the rat tail) was determined using blood glucose monitor Accu-Chek Advantage II (Roche Diagnostica, Sao Paulo, SP, Brazil). Animals were considered diabetic if glucose was above $200 \mathrm{mg} / \mathrm{dL}$. Insulin treated group, received 4 IU of neutral protamine Hagedorn insulin (Eli Lilly, São Paulo Brazil.) s.c., 8 hours before the experiment and other groups received saline solution.

\section{Bronchoalveolar lavage}

Animals were anesthetized by an intraperitoneal injection of ketamine hydrochloride $90 \mathrm{mg} /$ kg (Ceva Santé Animale, Paulínia-SP, Brazil) and xylazine hydrochloride (Ceva Santé Animale, PaulíniaSP, Brazil) $10 \mathrm{mg} / \mathrm{kg}$. After being anesthetized, trachea was exposed through a midline ventral incision of approximately $0.5 \mathrm{~cm}$ length in the neck. Bronchoalveolar lavage (BAL) was performed by instillation of 10 $\mathrm{mL}$ of PBS, at room temperature, through a polyethylene tube (1 $\mathrm{mm}$ in diameter) inserted into the trachea, as published previously [16]. First $10 \mathrm{~mL}$ instilled into the lungs was withdrawn and reinjected twice. After centrifugation ( $500 \mathrm{~g}$ for $10 \mathrm{~min}$ ), the supernatant was kept at $-70^{\circ} \mathrm{C}$ until cytokine measurements. The lungs were further lavaged with $15 \mathrm{~mL}$ PBS $(3 \times 5 \mathrm{~mL})$ to obtain remaining leukocytes. The BAL fluid was not used if the retrieved volume was less than $85 \%$ of the $25 \mathrm{~mL}$ instilled [16].

\section{Enzyme immunoassay for cytokines and chemokines}

The concentrations of tumor necrosis factor (TNF)- $\alpha$, interferon (INF)- $\gamma$, cytokine-induced neutrophil chemoattractant 1 (CINC-1) and CINC-2, interleukin (IL)-1 $\beta$, IL-10 and IL-6 in the BAL supernatant were determined by enzyme-linked immunosorbent assay (ELISA) using commercially available kits according to the manufacturer's instructions (R\&D Systems Inc, Minneapolis, USA). The sensitivity of the assay was $15 \mathrm{pg} / \mathrm{mL}[16]$.

\section{Bone Marrow derived Macrophages}

Bone marrow macrophages (BMM) were generated as published previously [17-19]. In summary, femur and tibia from rats were flushed with RPMI 1640 medium, centrifuged and resuspended $\left(10^{6}\right.$ cells/ml) in RPMI, enriched with 10\% heat-inactivated fetal calf serum (FCS) either with rat recombinant macrophage colony-stimulating factor (M-CSF) or granulocyte-macrophage colony-stimulating factor (GM$\mathrm{CSF}$ ) (both from Peprotech, Princeton, NJ, USA). On the $4^{\text {rd }}$ day of incubation in $5 \% \mathrm{CO}_{2}, 37^{\circ} \mathrm{C}$, nonadherent cells (BMM precursors) were collected and further incubated for 3 days with fresh medium enriched with the same growth factor (same as above). On day 7, adherent cells from the plates were harvested by vigorous pipetting and used for experiments. Some of the cultures were also performed on the top of coverslips (for microscopy).

\section{Histology and flow cytometry}

Spleens were either fixed in formaldehyde or snap frozen in Tissue-Tek OCT (Sakura, Torrance, CA, USA). Sections (8-10 $\mu \mathrm{m})$ were cut, air dried overnight and those not fixed with formaldehyde were fixed in cold acetone. Immunohistochemistry was performed as previously published [20]. Images were acquired using a Leica confocal laser scanning microscope (Leica Microsystems, GmBH, Wetzlar, Germany) equipped with 1 argon and 2 HeNe lasers, using magnification lens at 20×/0.70 CS and 63×/1.32 immersion oil and $90 \%$ glycerol (MP Biomedicals). The pictures are representative of at least 3 analysed spleen sections per rat and for BAL, triplicates from the same sample was performed. To quantify fluorescence, Integrated Density was determined by Fiji (Image J, NIH, USA). For flow cytometry analysis, single cell suspensions from BAL, BMM and spleens were prepared and erythrocytes lysed. Cells were immunolabelled and acquired using LSR Fortessa (Becton, Dickinson and Company, NJ, USA). Analyses were made using FlowJo software (version X.0 TreeStar Inc.) [20]. 
Antibodies

The following antibodies were used for flow cytometry and/or immunohistochemistry: CD11b/cPE-Cy7, Tcell $\alpha \beta$ PerCP, (BD Bioscience, NJ, USA); MHCII PE, anti-rabbit IgG FitC, CD45-APC, (eBioscience, SanDiego CA); anti-rabbit alexa555, anti-rabbit-Cy3, Anti-rat pan macrophage marker (KiM2R) (Abcam, Cambridge, UK); Live/Dead-AmCyan (Invitrogen), anti-rabbit APC-Cy7 (Santa Cruz Biotechnology); antiautophagy related protein (Atg) 3, 5, 7, 12, LC3A, B (Cell Signaling).

\section{Data and statistical analysis}

Data shown was analyzed by ANOVA or unpaired t-test using GraphPad Prism 6.0 software (La Jolla, CA, USA) and a two-tailed $p$ value with $95 \%$ confidence interval was acquired. Data is presented as mean (and \pm SEM) and $\mathrm{P}<0.05$ was considered significant.

\section{Results}

Higher expression of LC3 in macrophages from bronchoalveolar lavage in diabetic rats treated with insulin

Consistently with previous studies [16], ALX treated rats showed typical DMI signs, including elevated blood glucose and lower rate of weight gain (data not shown). LC3 is a soluble protein ubiquitously distributed in mammalian cells and tissues. Using a validated flow cytometry technique that excludes cytoplasmic form of LC3, we examined macrophages from BAL and screened for their LC3 autophagosomal membrane bound content (Fig. 1D). While AM from both control and diabetic rats did not show LC3 expression differences, AM from diabetic animals treated with insulin showed 5 fold enhanced LC3 autophagosomal membrane bound expression (Fig. 1D). Confocal studies were also performed (Fig. 2) and analysis of BAL cells integrated density showed higher accumulation of LC3 on cells derived from diabetic animal treated with insulin (Fig. 2 A). Cytokines from BAL were determined but only CINC-1 (Fig. 3A) and CINC-2 (Fig. 3B) expressions were detected. Interestingly,

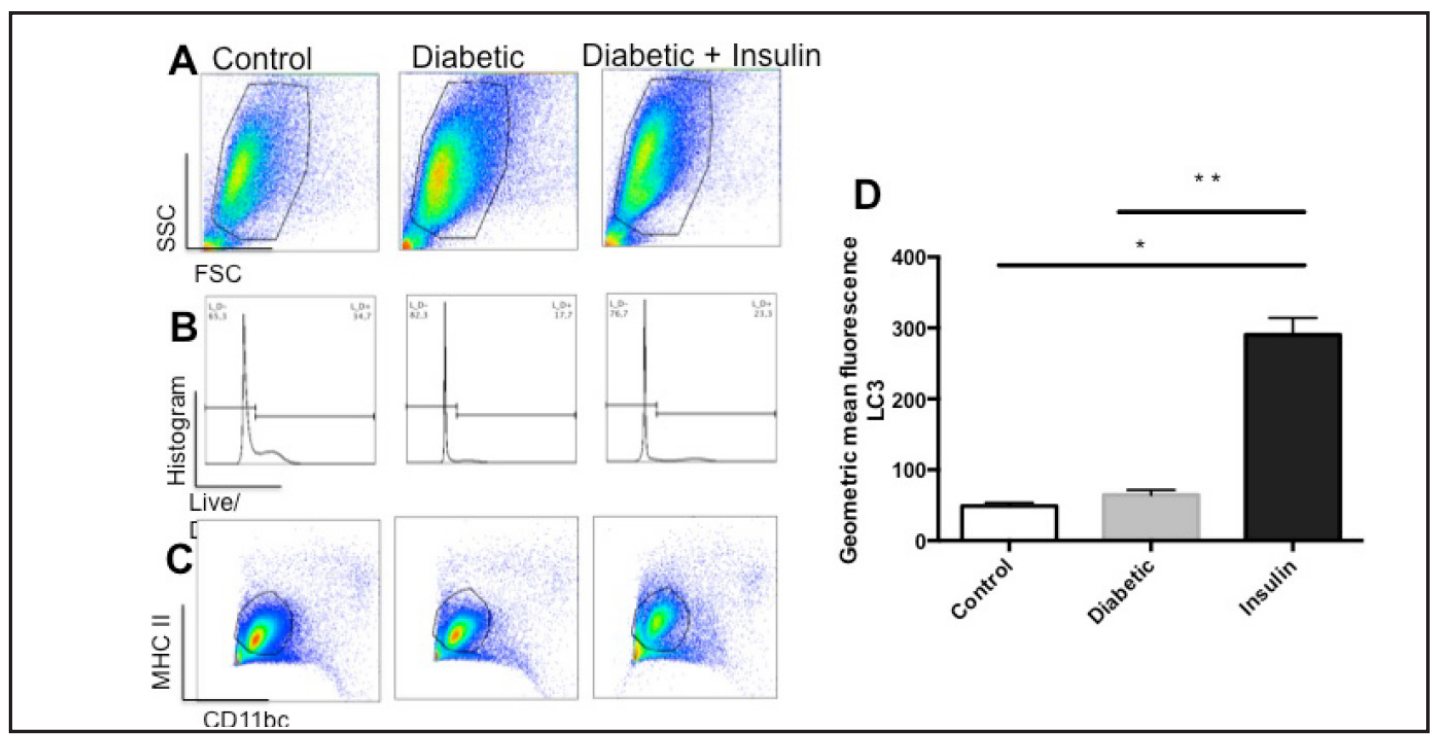

Fig. 1. Autophagosome expression of LC 3 in alveolar macrophages: BAL was performed by instillation of $10 \mathrm{~mL}$ of PBS, at room temperature and extra 3 times of $5 \mathrm{~mL}$ for total cell recovery. Fig. A, B and $\mathrm{C}$ show flow cytometry gatings. In A, BAL cells gated. In B, live cells were discriminated and in $\mathrm{C}$, macrophages were determined as being double positive for MHC II and CD 11bc. In D, LC3 geometric mean expression was measured on macrophages obtained from control, diabetic and insulin treated rats. Graph represents geometric mean fluorescence of LC3 \pm SEM. ( ${ }^{*}$ and $\left.{ }^{* *} \mathrm{p}<0.005\right)$ Representative of 3 independent experiments, 5 animals each group. 


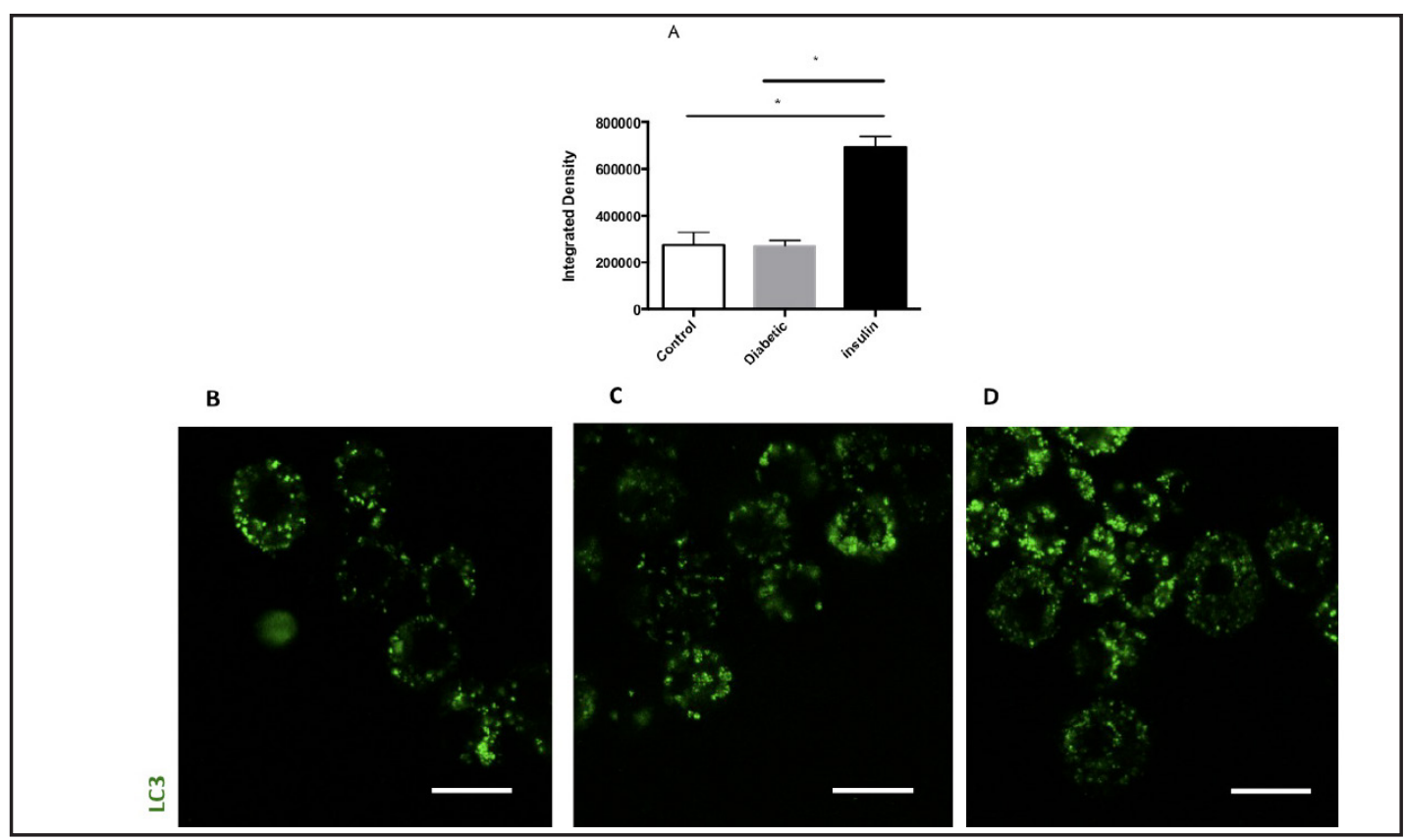

Fig. 2. Insulin enhances granularity of autophagosome LC3 in bronchoalveolar (BAL) macrophages: BAL was performed by instillation of PBS, at room temperature. BAL cells were recovered and LC3 integrated density measured on alveolar macrophages (AM) in (A). Graph representing Integrity density of LC3 \pm SEM. Below, 3 pictures of confocal microscopy showing LC3 staining (green channel) of AM from control (B), diabetic (C) and insulin treated (D) rats. Bars $=15 \mu \mathrm{m}$. Magnification $63 \mathrm{x}$, zoom 2. $(* \mathrm{p}<0.002)$.

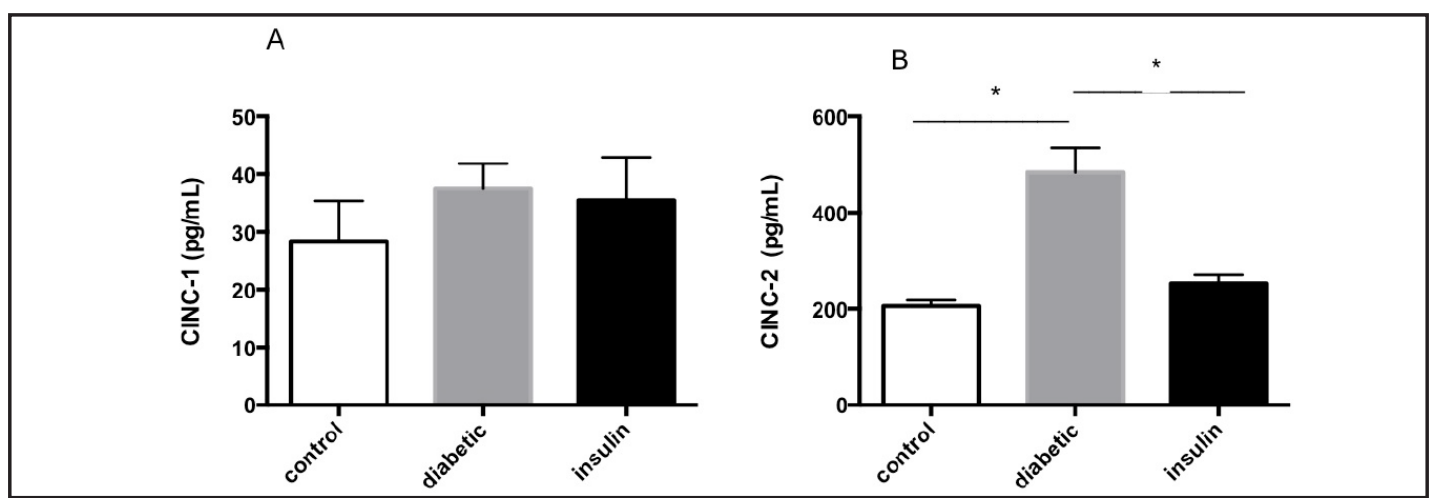

Fig. 3. CINC 1 and 2 in bronchoalveolar lavage (BAL): BAL was performed by instillation of PBS, at room temperature and levels of CINC 1 (A) and 2 (B) was measured in BAL from control, diabetic and insulin treated rats. Graphs represents concentration of the cytokines \pm SEM. ${ }^{*} \mathrm{p}<0.001$. Representative of 3 independent experiments, 6 animals each group.

treatment with insulin was able to restore CINC 2 to that of control rats. IL1 $\beta$, IL-6, TNF- $\alpha$, IL-10 and INF- $\gamma$ were measured, but their presence was below detectable values.

Distinct phenotypes in bone marrow derived macrophages from control and diabetic rats

To determine if environment can modulate LC 3 uptake in AM precursor cells, two lineages of macrophages called as M1 and M2 macrophage-like were generated from bone marrow progenitors. Bone marrow progenitor cells from control, diabetic and diabetic rats treated with insulin were isolated and cultured in RPMI with normal glucose concentration media. To assess activation of the autophagy pathway we determined the LC3 autophagosome bound content by flow cytometry. After one week in culture, M1-like macrophages from diabetic and diabetic rats treated with insulin showed lower LC3 contents, compared to control animals (Fig. 4D). Conversely, M2-like macrophages from both diabetic groups 


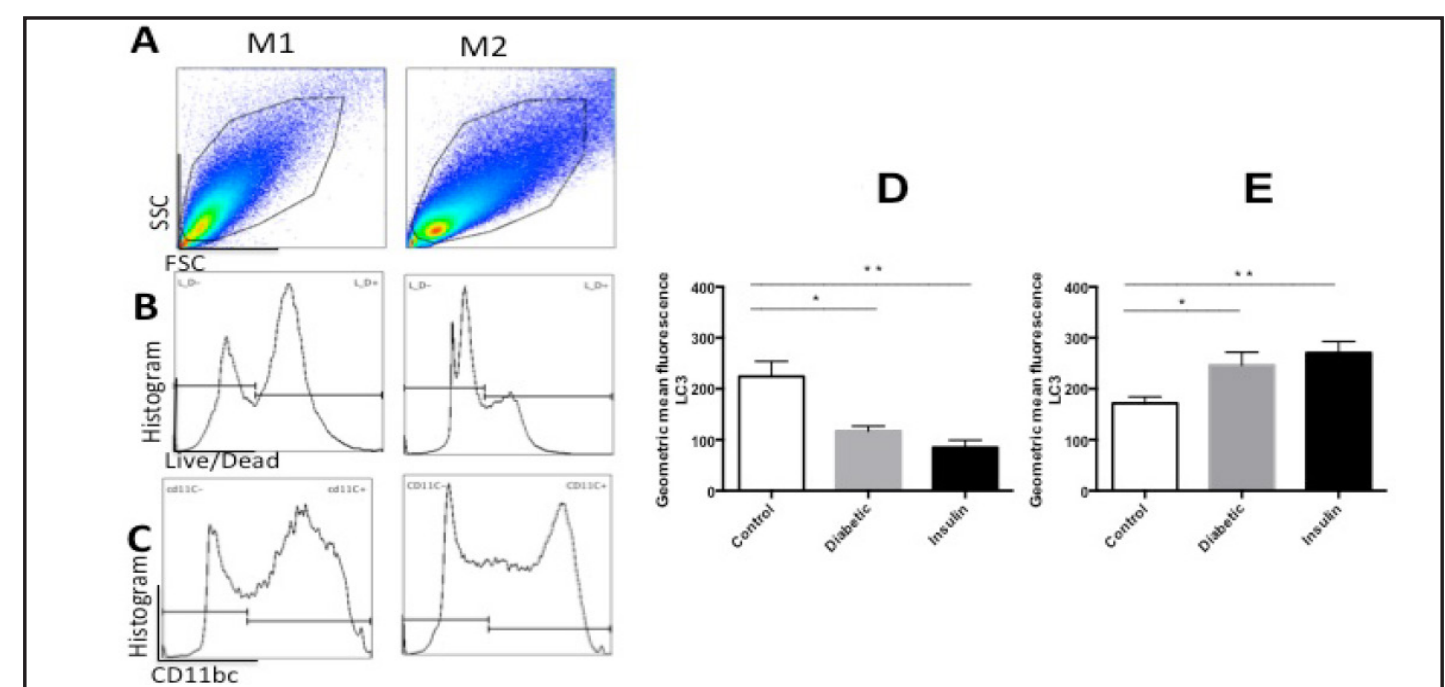

Fig. 4. Insulin Influence upon LC3 on Bone Marrow derived macrophages (BMM). Bone marrow from femur and tibia was processed and cultured for one week either with GM-CSF or M-CSF, generating both types Type I (M1), or Type 2 (M2)-like macrophages. Autophagy marker LC 3 content was screened by flow cytometry on (D) M1-like BMM and on (E) M2-like BMM derived from control, diabetic and insulin treated animals. Graphs represent geometric values of LC3 +/- SEM. 6 animals per group, representative of 3 independent experiments. ( $*$ and ${ }^{* *} \mathrm{p}<0.05$ on both graphs).

Fig. 5. M1 and M2 LC3 Integrated density: Bone marrow extracted from femur and tibia was cultured for one week either with GM-CSF or M-CSF, generating both: Type I (M1) and Type 2 (M2)-like macrophages. Confocal microscopy of M1 $\left(A_{1,2,3,4,5,6}\right)$ and M2 ( $\left.B_{1,2,3,4,5,6}\right)$ macrophages was performed. Odd numbers show grayscale pictures of the green channel (LC3) and even numbers show RGB pictures (red is KiM2R macrophage marker, green is LC3, and blue is CD45). Integrated density was calculated on both types of macrophages M1 (A) and M2 (B)-like macrophges, differentiated from control $(1,2)$, diabetic $(3,4)$ and insulin treated $(5,6)$ rats. Analysis of 6 animals in each group, at least 3 pictures of each culture per animal. One representative picture per animal from each group. Graphs represent mean integrated density values of LC3 $\left( \pm\right.$ SEM). ( ${ }^{*}$ and $* *$ $\mathrm{p}<0.05$ ) Bars $=15 \mu \mathrm{m}$. Magnification $63 x$, zoom $2 x$.
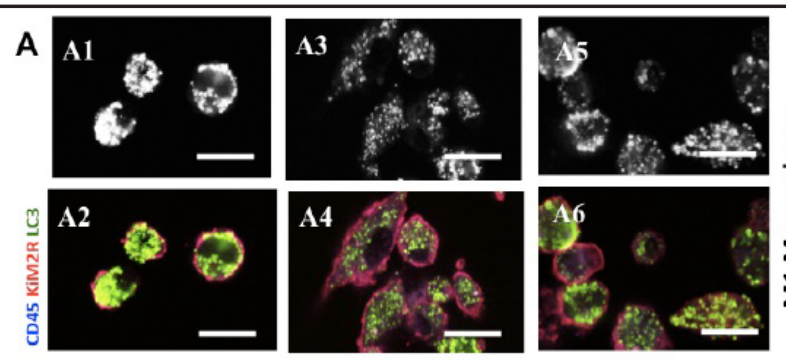

Insulin
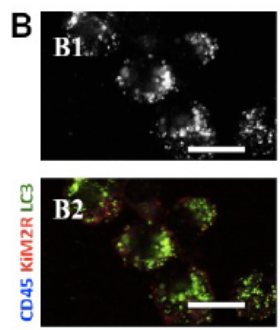

Control
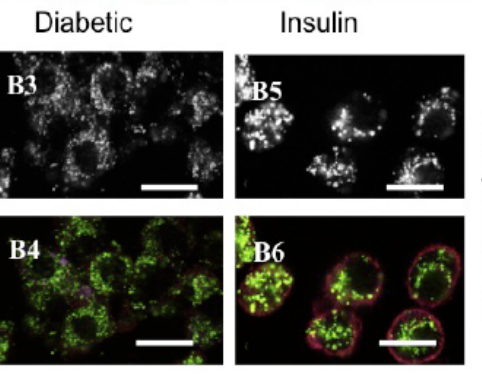

Insulin
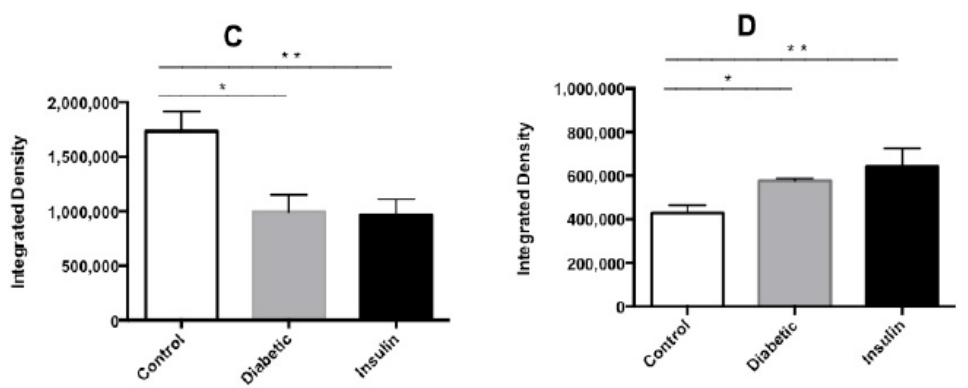
Fig. 6. Spleen autophagy levels: Spleens from normal, diabetic and insulin treated rats were frozen in OCT and sectioned. Confocal microscopy showing spleen histology (with follicles) in A, B, C (bar $=400 \mu \mathrm{m}$ magnification 20x) and red pulp macrophages in D,E,F (bar $=25 \mu \mathrm{m}$ magnification 63x zoom 1) from control (A,D), diabetic $(\mathrm{B}, \mathrm{E})$ and insulin treated $(\mathrm{C}, \mathrm{F})$ rats. Data presented as RGB pictures (red is KiM2R macrophage marker, green is LC3, and blue is CD45 Integrated density from Atg 12 content in spleen (G) red and $(\mathrm{H})$ white pulp $\left({ }^{*} \mathrm{p}<0.05 ;{ }^{* *} \mathrm{p}<\right.$ 0.001 ) was measured.

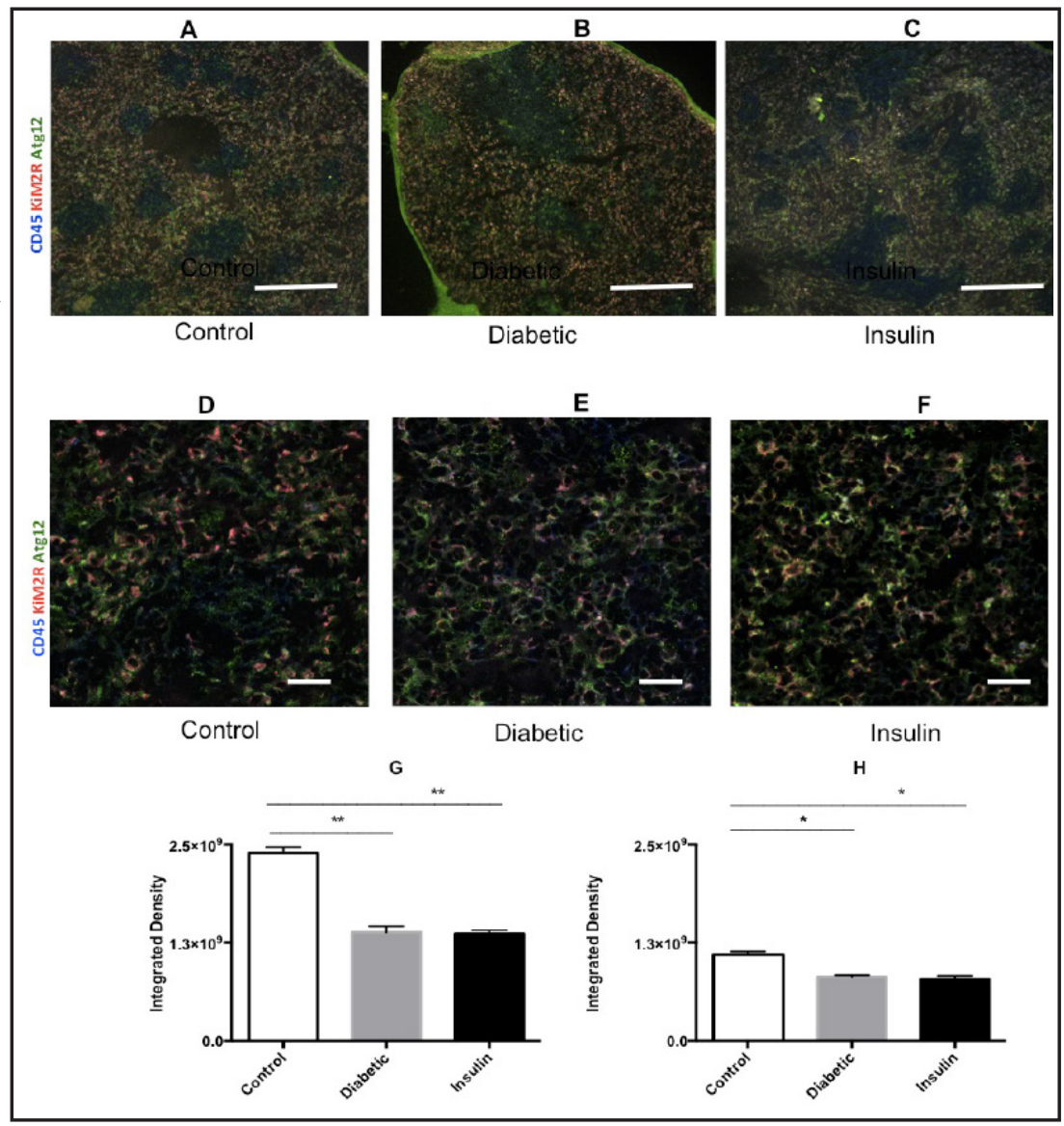

exhibited higher LC3 autophagosome bound levels (Fig. 4E) than healthy animals. When M1 and M2 BMM were screened by confocal microscopy and integrated density of LC3 measured, the same trend was found (Fig. 5 C, D). In Fig. 5 A and B we show images in RGB (red, green, blue) and gray scale of the green channel in BMM from control (Fig. 5 A1, B1); diabetic (Fig. 5 A3, B3); and diabetic animals treated with insulin (Fig. 5 A5, B5).

\section{Diabetic Macrophages in spleen showed lower levels of autophagy}

Spleens from control, diabetic and diabetic animals treated with insulin were examined for autophagy activation using Atg 12 (Fig. 6). When integrated density of red and white pulp was compared, macrophages showed higher autophagic content compared to other leucocytes (Fig. 6). After diabetic rats were treated with insulin, autophagy related marker did not recover basal levels (Fig. $6 \mathrm{G}, \mathrm{H}$ ).

\section{Discussion}

Data presented herein suggest that expression of the LC3 bound to autophagosomes, varies depending on the type of macrophage. Insulin seems to influence in multifaceted ways macrophages from distinct origins. In ALX induced diabetes in rats, hyperglycemia and absence of insulin did not change autophagosome LC 3 levels in BAL. When bone marrow cells were differentiated into M1-like macrophages, those cells derived from diabetic animals have lowered their autophagosome LC3 content. Conversely, cells differentiated into M2like macrophages, have their autophagic LC3 content enhanced. When one of the proteins (Atg12) responsible for conjugating LC3 into the phagosome was screened, it was diminished in the splenic macrophages (SM) from red pulp of the diabetic animals, compared to healthy control. When diabetic rats were treated with insulin, SM failed to restore Atg 12 content. 
Higher prevalence of infection contributes to an elevated mortality in diabetic patients $[21,22]$. Many mechanisms can relate predisposition to infection in diabetes and dysfunction of the innate immunity is considered one of the major explanations [23]. Important for antigen processing, AM are responsible for respiratory infections associated to DMI [11, 24]. Most of the experimental literature has suggested that phagocytosis in macrophages and antigen presentation to $\mathrm{T}$ cells is impaired $[11,25]$. The autophagy pathway has been less studied in diabetic macrophages. Being important to maintain homeostasis, autophagy pathway imbalance in macrophages has been associated to non-infectious $[26,27]$ and infectious [5, 28] diseases. Manipulation of defects in autophagic signalling has been shown to attenuate glomerular damage [29] and ameliorate cardiac injury in mice [30]. Since insulin is a modulator of the phosphatidylinositol 3 kinase (PI3K) and mammalian target of rapamycin (mTOR) pathway [5], DMI is a natural model for autophagy pathway imbalance. Here, with the ALX induced rat model, we studied autophagy markers in different sources of macrophages from a non-infectious perspective.

Previous studies of our group have suggested that using this animal model, AM showed impaired phagocytosis [11] and the treatment with insulin restored their function. Also, some inflammatory parameters such as cytokines expression (IL-6, CINC-1 and CINC-2) were restored by insulin [13]. Since phagocytic pathway also requires LC3 recruitment, we have chosen to study the LC3 expression in these cells. LC3 as shown recently, counteracts in both processes: phagocytosis [31] and autophagy [31,32]. It has been also demonstrated that LC3 can be recruited to the phagosome, constituted by a single membrane in a process named LC3 associated phagocytosis (LAP) [33]. LAP is a process distinct from autophagy and is always related to pathogen engulfment. During autophagy, a lipidated form of the LC3, required for membrane elongation, is not necessarily dependent on the presence of a pathogen. Autophagy is a continuous process needed for turnover of proteins and organelles [5]. There are many LC3 isoforms and the autophagy hallmark indicator is the lipidated membrane-bound form LC3II [32]. Most of previous studies have used Western blotting to determine levels of LC3II expression. Unfortunately this method is not the best to use, since BAL is constituted by an assortment of cells. Recently, the Autophagy journal has accepted the use of LC3 II as an autophagy marker [34]. Therefore, we used a flow cytometry protocol for autophagy [35] and combined the usage of LC3B with markers for macrophages. Our results showed that LC3 protein is less expressed on AM vesicles from diabetic rats compared to those treated with insulin. Intriguingly, levels of LC3 from diabetic AM were the same as found in control rats. It was not possible to affirm if diabetic AM fail to upregulate autophagy or the absence of insulin and associated hyperglycemia induce AM to protect the host by not enhancing the autophagy as a beneficial adaptive process [30]. When insulin was added to the system, AM started to enhance LC3 content. Despite not having developed functional assays, this result suggested that AM are not impaired, they are able to perform autophagy, but they refrain themselves probably, as inferred previously, to self protecting. A recent published review about autophagy in skeletal muscle suggested that in glycogen storage diseases, the accumulation of autophagosomes along the myofibers indicated a pathologic phenotype [36] and this is in accordance to previous phenomena observed in cardiomyocytes.

Spleen macrophages and cells from white pulp of the diabetic rats showed lower Atg 12 protein expression. When insulin was administrated, these cells did not restore previous levels of Atg 12. It is intriguing because insulin was capable of restoring AM phagocytosis [11]. Why insulin enhanced LC3 levels in AM but not in SM remains unclear. However, if AM have their phagocytic function restored by insulin and are capable of increasing LC3 levels, maybe AM have not impaired function. Up to date, it is not known whether there is a hierarchy relating triggering stimulus and AM response. Also, all macrophages types were not screened and certainly one type of macrophage may suffer from the absence of insulin more than the others. What we would like to highlight here is that there might exist a signaling hierarchy that was not fully understood. During inflammation, in diabetic hosts, when insulin cannot exert its function, some immune cells may have a shut down system to protect the host. However, the protection turns against the organism, if sustained for long 
time. Maybe, the inflammatory and infectious signaling cannot overcome the absence of insulin during a certain period. A review by Rabinowitz and White, 2010, mentioned that the energy consumption with autophagy is approximately $1 \%$ of the total amount of energy necessary for protein synthesis each hour [37]. Probably, this amount of energy cannot be lost for performing autophagy when there is a lack of insulin, and difficulty in bringing glucose from the outside to the inner part of the cell. Therefore, immune cells may fail to enhance turn over of self-damaged organelles in order to save energy. Low autophagy levels in diabetic AM are in accordance with the concomitant diminished phagocytosis, since phagocytosis and autophagy although different, are related pathways.

Regarding BMM, hyperglycemia and insulin absence acts antagonistically in M1 and M2 macrophages. Pro inflammatory M1 macrophages had their LC3 content decreased in the absence of insulin, while M2 macrophages exhibited higher amount of LC3. M1 and M2 nomenclature was adopted in analogy to Th1 and Th2 T cell responses. As Sica and Mantovani described [38], the better understanding of the polarization M1-M2 provides knowledge for macrophage-centered diagnosis, helping to develop treatment based on innate immunity as a target.

Since autophagy is related to the origin of energy production and expenditure, a better understanding of the whole pathway is required. We strongly believe that discovering an alternative to manipulate the autophagy pathway in favor of diabetic patients, it will be possible to control many of the infections since it might recover phagocytic function although functional assays should be performed in diabetic patients.

\section{Acknowledgments}

The authors would like to sincerely thank Irene M. Gouveia, Iger Bodin and Mariana C.F. Silva for their expert technical help. The authors are supported by grant 2010/02272-0 from São Paulo Research Foundation (FAPESP), grant 470523/2013-1 from National Counsel of Technological and Scientific Development (CNPq, Projeto Universal 2013) Coordenação de Aperfeiçoamento de Pessoal de Nível Superior (CAPES) and Pró-reitoria de Pesquisa da Universidade de São Paulo (PRP/USP, Projeto I and Novos Docentes), Brazil. The authors declare that there is no conflict of interest that would prejudice the impartiality of this scientific work.

\section{References}

Wolfe RR: Effects of insulin on muscle tissue. Curr Opin Clin Nutr Metab Care 2000;3:67-71.

Zeigerer A, Lampson MA, Karylowski O, Sabatini DD, Adesnik M, Ren M, McGraw TE: GLUT4 retention in adipocytes requires two intracellular insulin-regulated transport steps. Mol Biol Cell 2002;13:2421-2435.

-3 Gonzalez CD, Lee MS, Marchetti P, Pietropaolo M, Towns R, Vaccaro MI, Watada H, Wiley JW: The emerging role of autophagy in the pathophysiology of diabetes mellitus. Autophagy 2011;7:2-11.

4 Nixon RA: Autophagy, amyloidogenesis and Alzheimer disease. J Cell Sci 2007;120:4081-4091.

-5 Levine B, Mizushima N, Virgin HW: Autophagy in immunity and inflammation. Nature 2011;469:323-335.

6 Yuk JM, Yoshimori T, Jo EK: Autophagy and bacterial infectious diseases. Exp Mol Med 2012;44:99-108.

7 American Diabetes association, 2014, last visit june 19 2014, http://www.diabetes.org/living-with-diabetes/ treatment-and-care/medication/other-treatments/flu-and-pneumonia-shots.html. Last Reviewed: June 7, 2013 Last Edited: April 7, 2014.

8 Sugawara I, Mizuno S: Higher susceptibility of type 1 diabetic rats to Mycobacterium tuberculosis infection. J Exp Med 2008;216:363-370.

9 Wertman KF, Henney MR: The effects of alloxan diabetes on phagocytosis and susceptibility to infection. J Immunol 1962;89:314-317.

10 Kane WC: Phagocytosis and Its Relationship to Infection in Diabetes Mellitus. Diabetes 1964;13:424.

-11 Ferracini M, Martins JO, Campos MR, Anger DB, Jancar S: Impaired phagocytosis by alveolar macrophages from diabetic rats is related to the deficient coupling of LTs to the Fc gamma R signaling cascade. Mol Immunol 2010;47:1974-1980. 
12 Mowat A, Baum J: Chemotaxis of polymorphonuclear leukocytes from patients with diabetes mellitus. N Engl J Med 1971;284:621-627.

13 Sunahara KK, Sannomiya P, Martins JO: Briefs on insulin and innate immune response. Cell Physiol Biochem 2012;29:1-8.

14 Gutierrez MG, Master SS, Singh SB, Taylor GA, Colombo MI, Deretic V: Autophagy is a defense mechanism inhibiting BCG and Mycobacterium tuberculosis survival in infected macrophages. Cell 2004;119:753-766.

-15 Bonilla DL, Bhattacharya A, Sha Y, Xu Y, Xiang Q, Kan A, Jagannath C, Komatsu M, Eissa NT: Autophagy regulates phagocytosis by modulating the expression of scavenger receptors. Immunity 2013;39:537-547.

-16 Martins JO, Zanoni FL, Martins DO, Coimbra R, Krieger JE, Jancar S, Sannomiya P: Insulin regulates cytokines and intercellular adhesion molecule-1 gene expression through nuclear factor-kappaB activation in LPS-induced acute lung injury in rats. Shock 2009;31:404-409.

17 Lari R, Fleetwood AJ, Kitchener PD, Cook AD, Pavasovic D, Hertzog PJ, Hamilton JA: Macrophage lineage phenotypes and osteoclastogenesis--complexity in the control by GM-CSF and TGF-beta. Bone 2007;40:323-336.

18 Fleetwood AJ, Lawrence T, Hamilton JA, Cook AD: Granulocyte-macrophage colony-stimulating factor (CSF) and macrophage CSF-dependent macrophage phenotypes display differences in cytokine profiles and transcription factor activities: implications for CSF blockade in inflammation. J Immunol 2007; 178:5245-5252.

19 Weischenfeldt J, Porse B: Bone Marrow-Derived Macrophages (BMM): Isolation and Applications. CSH Protoc 2008;pdb.prot5080.

20 Westerberg LS, Dahlberg C, Baptista M, Moran CJ, Detre C, Keszei M, Eston MA, Alt FW, Terhorst C, Notarangelo LD, Snapper SB: Wiskott-Aldrich syndrome protein (WASP) and N-WASP are critical for peripheral B-cell development and function. Blood 2012;119:3966-3974.

21 Roglic G, Unwin N, Bennett PH, Mathers C, Tuomilehto J, Nag S, Connolly V, King H: The burden of mortality attributable to diabetes: realistic estimates for the year 2000. Diabetes Care 2005;28:2130-2135.

22 Sarnak MJ, Jaber BL: Mortality caused by sepsis in patients with end-stage renal disease compared with the general population. Kidney Int 2000;58:1758-1764.

23 Lambrecht BN: Alveolar macrophage in the driver's seat. Immunity 2006;24:366-368.

24 Ma H, Liu G, Ding W, Wu Y, Cai L, Zhao Y: Diabetes-induced alteration of F4/80+ macrophages: a study in mice with streptozotocin-induced diabetes for a long term. J Mol Med 2008;86:391-400.

25 Glass EJ, Stewart J, Weir DM: Altered immune function in alloxan-induced diabetes in mice. Clin Exp Immunol 1986;65:614-621.

26 Deretic V, Saitoh T, Akira S: Autophagy in infection, inflammation and immunity. Nat Rev Immunol 2013;13:722-737.

27 Ryter SW, Cloonan SM, Choi AM: Autophagy: a critical regulator of cellular metabolism and homeostasis. Mol Cells 2013;36:7-16.

28 Campbell GR, Spector SA: Vitamin D inhibits human immunodeficiency virus type 1 and Mycobacterium tuberculosis infection in macrophages through the induction of autophagy. PLoS Pathog 2012;8:e1002689.

29 Fang L, Zhou Y, Cao H, Wen P, Jiang L, He W, Dai C, Yang J: Autophagy attenuates diabetic glomerular damage through protection of hyperglycemia-induced podocyte injury. PLoS One 2013;8:e60546.

-30 Xu X, Kobayashi S, Chen K, Timm D, Volden P, Huang Y, Gulick J, Yue Z, Robbins J, Epstein PN, Liang Q: Diminished autophagy limits cardiac injury in mouse models of type 1 diabetes. J Biol Chem 2013;288:1807718092 .

-31 Lai SC, Devenish RJ: LC3-Associated Phagocytosis (LAP): Connections with Host Autophagy. Cells 2012;1:396408.

32 Klionsky et al.: Guidelines for the use and interpretation of assays for monitoring autophagy. Autophagy 2012;8:445-544.

-33 Florey O, Kim SE, Sandoval CP, Haynes CM, Overholtzer M: Autophagy machinery mediates macroendocytic processing and entotic cell death by targeting single membranes. Nat Cell Biol 2011;13:1335-1343.

-34 Pei J, Zhao M, Ye Z, Gou H, Wang J, Yi L, Dong X, Liu W, Luo Y, Liao M, Chen J: Autophagy enhances the replication of classical swine fever virus in vitro. Autophagy 2014;10:93-110.

-35 Eng KE, Panas MD, Karlsson Hedestam GB, McInerney GM: A novel quantitative flow cytometry based assay for autophagy. Autophagy 2010;6:634-641.

-36 Neel BA, Lin Y, Pessin JE: Skeletal muscleautophagy: a new metabolic regulator. Trends Endocrinol Metab 2013;24:635-643.

-37 Rabinowitz JD, White E: Autophagy and metabolism. Science 2010;330:1344-1348.

-38 Sica A, Mantovani A: Macrophage plasticity and polarization: in vivo veritas. J Clin Invest 2012;122:787-795. 